\title{
Organisations de l'économie sociale et solidaire : quelle théorie de la gouvernance ?
}

\author{
Jean-Baptiste Cartier (*), Philippe Naszalyi(**) et Benoît PigÉ(***) \\ (C) 29 OCTOBRE 2012 \\ (*)Maître de Conférences en Sciences de Gestion \\ IAE de Lyon 3 - Centre de recherche Magellan - Finance \\ E-Mail : jean-baptiste.cartier@univ-lyon3.fr \\ (**)Professeur associé à l'Université d'Evry Val d'Essonne (L@REM) \\ Directeur de LaRSG \\ revue-sciences-gestion@wanadoo.fr \\ (***)Professeur des Universités en Sciences de Gestion \\ LEG-Fargo / Université de Franche-Comté \\ Email : benoit.pige@univ-fcomte.fr
}

Résumé : Un des enjeux des coopératives est de devenir les Organisations de référence dans l'application du modèle des parties prenantes tout en étant viables économiquement. Ceci suppose de résoudre les trois points suivants: assurer une gestion efficiente des ressources qui permette de réaliser des transactions à un coût inférieur à celui qui aurait lieu sur le marché ; mettre en œuvre des mécanismes de gouvernance permettant l'expression et la prise en compte des intérêts des diverses parties-prenantes ; mettre en œuvre des mécanismes de gouvernance permettant d'évaluer ex post l'action des dirigeants et la réponse apportée aux attentes des parties prenantes.

Abstract: A major aim for cooperatives is to become the model of stakeholders' organizations while keeping profitable. This supposes to resolve the three following points: to ensure an efficient management of resources which makes it possible to carry out transactions at a cost lower than that which would take place on the market; to design mechanisms of corporate governance allowing expression and implementation of stakeholders' interests and expectations; to implement mechanisms of control allowing to evaluate the action of top executives, particularly regarding satisfaction of expressed stakeholders expectations. 
Avec la crise économique générée par les excès du capitalisme financier, les Organisations de l'économie sociale et solidaire semblent retrouver un certain attrait et offrir un modèle de développement économique alternatif. La prise en compte d'objectifs complémentaires à celui de maximisation du profit apparaît ainsi comme une réponse aux attentes des citoyens.

Pourtant, d'un point de vue théorique, l'alternative, entre entreprises maximisant le profit et entreprises mutualistes ou coopératives, n'est pas nouvelle et le débat semblait tranché depuis les années 1960 et 1970 (M.C. Jensen et W.H. Meckling, 1979). Du point de vue de l'efficience économique des Organisations, les entreprises qui s'assignent comme objectif la maximisation du profit favorisent une plus grande création de valeur, et ainsi contribuent à un équilibre général optimal pour tous les acteurs économiques en présence de marchés efficients.

Cet article est structuré en trois parties. Dans la première, nous proposons une analyse théorique des modèles économiques qui permettent d'expliquer l'intérêt et les limites des structures coopératives. Dans une deuxième partie, nous appliquons notre analyse à la comparaison des banques mutualistes françaises et des banques classiques à capitaux. Dans la troisième partie, nous analysons plus spécifiquement la gouvernance d'une structure mutualiste (la Fédération Nationale Interprofessionnelle des Mutuelles). Notre démarche part donc d'une analyse générale pour aboutir à l'analyse d'un cas spécifique permettant de faire ressortir de façon pratique les enjeux théoriques de la gouvernance des mutuelles et coopératives.

\section{Les modèles théoriques}

Nous proposons d'effectuer une relecture de l'organisation des coopératives à la lumière de trois principales théories des Organisations : la théorie des coûts de transaction, la théorie de l'agence et la théorie des parties prenantes. 


\subsection{Le modèle coopératif comme alternative au marché dans le cadre de la théorie des coûts de transaction}

Dans son article de 1937, Coase recherchait une explication à l'existence d'Organisations en présence de marchés. En effet, par le biais de contrats de marchés il est possible de concevoir, produire et distribuer tous les produits ou services imaginables. C'est d'ailleurs une des leçons de l'économie Internet où l'on voit émerger des acteurs dont la seule fonction est de mettre en relation des acteurs économiques et de faciliter la création de mécanismes contractuels de marché.

Selon Coase, l'Organisation se différencie du marché par sa capacité à réaliser des transactions en interne à un coût de transaction moindre que celui qui serait supporté sur le marché. Cette différence dans les coûts de transaction supportés s'explique par les mécanismes de coordination et de contrôle mis en œuvre pour réaliser les transactions et en assurer le suivi dans le temps. Par le mécanisme de contrôle hiérarchique, les Organisations peuvent gérer des transactions et imposer des solutions sans qu'il y ait nécessairement un processus de négociation. De surcroît, en cas de difficulté dans l'exécution du contrat ou de la transaction, l'autorité hiérarchique peut départager les acteurs sans intervention d'un processus arbitral complexe.

Williamson $(1975,1985)$ a apporté une contribution déterminante en énonçant les caractéristiques des transactions qui justifient leur internalisation par rapport aux transactions qui peuvent être externalisées sur le marché (E. Ebondo et B. Pigé, 2002).

La question de la place des Organisations de l'économie sociale et solidaire est donc avant tout une question pragmatique si l'on se place du point de vue de la théorie des coûts de transaction : une coopérative permet-elle de réaliser une transaction donnée à un coût moindre que le marché et qu'une entreprise maximisant le profit (que nous dénommerons entreprise capitaliste pour la place prépondérante qui occupe le capital).

Ainsi, le fait que dans une entreprise coopérative certains acteurs occupent une double fonction: clients (ou fournisseurs ou salariés, selon la nature de la coopérative) et coopérateurs, c'est-à-dire détenteurs d'un droit de vote et de décision en assemblée générale, 
peut permettre la résolution de transactions à un coût moindre car l'asymétrie d'information et la divergence d'intérêts peuvent être plus faibles que dans une entreprise capitaliste, ce qui réduit les besoins de contrôle.

Inversement, une certaine dilution des incitations peut favoriser les comportements dits de passager clandestin (« free rider» en anglais) puisque quelques acteurs peuvent chercher à bénéficier d'avantages au détriment de la collectivité des acteurs, que ce soit en travaillant moins, ou en se contentant d'une qualité de production ou de service inférieure à la moyenne.

Dans le cadre théorique des coûts de transaction, l'enjeu de la gouvernance des coopératives est d'identifier les mécanismes de gouvernance qui offrent un avantage comparatif au modèle coopératif.

\subsection{Le modèle coopératif du point de vue de la théorie de l'agence}

La théorie de l'agence adopte un point de vue très différent mais complémentaire de la théorie des coûts de transaction. L'enjeu n'est plus de comprendre en quoi et comment les Organisations permettent de gérer certaines transactions à un coût moindre que les marchés, mais de réduire les coûts liés à la gouvernance des Organisations. Pour la théorie de l'agence, des coûts apparaissent dès lors qu'il y a une dissociation entre la gestion de l'Organisation et la perception des revenus résiduels qui découlent de cette gestion. Il est alors possible de déterminer une hiérarchie entre les différents modes de gouvernance possibles selon les coûts d'agence que chacun d'entre eux génère. La théorie de l'agence n'ignore pas qu'il existe des facteurs de contingence qui expliquent l'apparition et le développement de la dissociation des fonctions de direction, de contrôle et de propriété (au sens de perception des revenus résiduels), mais elle se focalise sur les mécanismes et les institutions à mettre en œuvre pour réduire les coûts d'agence liés à cette dissociation (G. Charreaux, 1987).

A la suite de Jensen et Meckling (1976), l'objectif principal de la théorie positive de l'agence est devenu l'alignement de l'intérêt des dirigeants sur celui des propriétaires. Ce rôle central des propriétaires était lui-même dû à la conviction (A.A. Alchian et H. Demsetz, 1972) que, dans un travail collectif, il est nécessaire d'avoir un agent qui perçoive les revenus 
résiduels et qui exerce la fonction de contrôle pour que l'on puisse aboutir à une gestion efficiente des ressources (E.F.Fama, 1980 ; E.F.Fama et M.C. Jensen, 1983).

Transposé au modèle coopératif, la première difficulté consiste à définir les propriétaires de l'entreprise coopérative, c'est-à-dire ceux qui en perçoivent les revenus résiduels. Et là, apparaît une divergence forte entre :

- Les coopérateurs pour une année $\mathrm{N}$ : qui peuvent percevoir une fraction des revenus résiduels sous forme de ristournes.

- Les coopérateurs ultérieurs : qui bénéficient des fruits du réinvestissement des revenus résiduels non distribués en année $\mathrm{N}$.

Alors que dans une entreprise capitaliste, par le biais du droit de propriété qui peut être vendu sur le marché, il existe une convergence d'intérêt entre les actionnaires de l'année $\mathrm{N}$ et les actionnaires ultérieurs (que ce soit les mêmes ou non), il n'en est pas de même dans l'entreprise coopérative. Si une fraction des coopérateurs ultérieurs n'était pas présente en année $\mathrm{N}$, ceux-ci n'en bénéficient pas moins des revenus résiduels capitalisés par les coopérateurs en année N. Il existe donc un transfert de richesse dans le temps qui ne se matérialise pas dans la richesse de chacun des coopérateurs, considérés individuellement.

Une deuxième divergence tient au mode de représentation des coopérateurs, c'est-à-dire le principe démocratique, un homme- une voix. Ce mode de représentation ne permet pas, à l'inverse du principe capitalistique, une action - une voix, de rendre compte des variations dans l'intérêt économique porté par les différents coopérateurs (Collette et Pigé, 2008). Qu'un coopérateur ait contribué à $10 \%$ de l'activité d'une coopérative ou à seulement $0,01 \%$, il bénéficiera du même droit de vote et du même pouvoir de décision en assemblée générale (avec parfois quelques modulations possibles qui restent néanmoins marginales).

Ces deux difficultés conjuguées ensemble soulèvent un véritable problème dans l'identification de la relation d'agence. S'il est bien possible d'identifier un agent (les dirigeants) et un principal (les coopérateurs), il n'est néanmoins pas possible de déterminer un objectif qui serait commun à la totalité des coopérateurs. En effet, dans le cadre de 
l'entreprise capitaliste, le principe de maximisation du profit est indissolublement lié au fait que la répartition temporelle du profit n'influera pas sur la richesse individuelle de chaque actionnaire puisque ce dernier peut toujours céder son action pour maximiser son intérêt économique temporel. Ce n'est pas le cas pour un coopérateur puisque la valeur de la part coopérative détenue est indépendante de la valeur de marché de l'entreprise coopérative. De surcroît, la répartition des revenus résiduels, si elle tient compte de l'activité économique réalisée par chaque coopérateur avec l'entreprise coopérative, ne le fait que dans un cadre annuel sans prendre en compte le volume des activités économiques réalisé au cours des années antérieures.

La théorie de l'agence permet donc de souligner une difficulté majeure de la gouvernance des entreprises coopératives : l'absence de critère de performance objectif sur lequel évaluer l'action de l'agent, c'est-à-dire des dirigeants.

Dans la tentative de réduire les coûts d'agence entre le principal et l'agent, entre les coopérateurs (les actionnaires dans le cas d'une entreprise classique) et les dirigeants, la théorie de l'agence propose de nombreux mécanismes de contrôle visant soit à réduire l'asymétrie d'information entre le principal et l'agent (ce qui permet au principal d'évaluer en connaissance de cause les actions et les décisions de l'agent), soit à aligner l'intérêt de l'agent sur celui du principal. Comme nous l'avons souligné, l'intérêt du principal ne peut pas être aisément défini dans une entreprise coopérative dès lors que l'on tient compte de la dimension temporelle et, par conséquent, chercher à aligner l'intérêt des dirigeants sur celui des coopérateurs est, sinon impossible, du moins extrêmement complexe à mettre en œuvre.

Mais la mise en œuvre de mécanismes de contrôle visant à réduire l'asymétrie d'information n'est pas pour autant plus simple. Les mécanismes habituellement proposés par la théorie de l'agence sont: le reporting comptable et financier, l'audit, et la spécialisation d'un ou plusieurs actionnaires qui acceptent d'assumer une fonction plus lourde de contrôle des dirigeants (qu'ils arrivent à rentabiliser en raison de la participation au capital qu'ils détiennent dans l'entreprise et de leur capacité à se faire entendre en assemblée générale et à être représentés au sein du conseil d'administration) (B. Pigé, 2008). 
Cette dernière possibilité est moins effective pour les entreprises coopératives car les coopérateurs les plus actifs ne détiennent pas une représentation en assemblée générale proportionnelle à leur activité réalisée avec la coopérative. Par conséquent, s'ils peuvent chercher à influencer la gestion de la coopérative, ils ne le feront pas (ou rarement) à travers des interventions en assemblée générale (qui auraient pour objet de discipliner ou de contrôler les dirigeants), mais plutôt par des relations directes avec les dirigeants en vue d'améliorer la gestion des transactions qu'ils réalisent avec la coopérative (améliorations qui peuvent bénéficier aux autres coopérateurs mais sans que cela soit l'objectif recherché). Alors que les actionnaires principaux ont un intérêt direct à améliorer à long terme l'efficience dans la gestion des ressources de l'entreprise (car cette efficience se traduit dans la valorisation de leurs actions), les coopérateurs ont un intérêt limité à leur horizon de coopération.

En ce qui concerne le reporting comptable et financier, il existe également une divergence insuffisamment prise en compte par la théorie de l'agence, en grande partie négligée par la normalisation comptable internationale (B. Pigé et X. Paper 2009), et qui porte sur la nature de l'information comptable diffusée. En effet, le cadre conceptuel de l'IASB s'oriente de plus en plus nettement vers la fourniture d'une information financière utile à la prise de décision pour les apporteurs de capitaux et les créanciers. Or, dans une entreprise coopérative, les coopérateurs ne sont pas d'abord des apporteurs de capitaux mais avant tout des apporteurs d'activité économique (par les biens ou les services qu'ils acquièrent, par ceux qu'ils fournissent, ou par leur travail). L'information pertinente, pour eux, n'est pas nécessairement une information financière. Se pose alors une réelle question sur la nature de l'information à transmettre aux coopérateurs et, par voie de conséquence, sur la nature de la mission de contrôle à conférer aux auditeurs. Ceux-ci doivent-ils limiter leurs diligences à la seule information comptable et financière, ou leurs diligences doivent-elles s'étendre aux autres formes d'information? 


\subsection{Les enjeux du modèle coopératif à la lumière de la théorie des parties prenantes}

La théorie des parties prenantes est une théorie actuellement inachevée car, à quelques exceptions près (dont B. Pigé 2010), elle ne permet pas de définir précisément les parties prenantes, leurs droits et leurs devoirs, ni leur implication dans la gouvernance de leur Organisation. Néanmoins, certains traits généraux sont considérés comme constituant cette théorie :

- Dans cette approche, les intérêts de toutes les parties prenantes doivent être pris en compte en tant que fins et non pas uniquement en tant que moyens.

- La primauté de l'éthique sur la recherche du profit.

Selon ces critères, le modèle coopératif offre d'indéniables avantages par rapport au modèle purement capitaliste. En effet, l'incapacité à définir un objectif unique pour tous les coopérateurs et la nécessité de parvenir à un compromis est, en soi-même, un facteur clé de prise en compte de la diversité des attentes. De surcroît, le fait que les coopérateurs soient à la fois clients (ou fournisseurs ou employés) et collectivement propriétaires de leur entreprise, introduit nécessairement la prise en compte d'un intérêt plus large que celui des seuls détenteurs de parts.

Mais, en même temps, cette théorie souligne les limites du modèle coopératif. Si les dirigeants doivent effectivement servir plusieurs maîtres, ils se trouvent confrontés à diverses alternatives :

- N'en servir aucun, en jouant sur les divisions entre les coopérateurs, sur la difficulté à mettre en œuvre un contrôle effectif, sur certaines pratiques d'autosélection des administrateurs.

- N'en servir que quelques-uns: les dirigeants peuvent privilégier certaines relations avec une catégorie de coopérateurs sur la base d'un échange de prestations privilégiées en contrepartie d'un soutien en assemblée générale ou en conseil d'administration. 
- Ne servir que les coopérateurs : en considérant les autres parties prenantes comme des moyens pour maximiser la satisfaction des coopérateurs, que cette dernière soit la maximisation du profit ou la maximisation des ristournes reversées ou des prix perçus.

- Servir la coopérative dans le respect des intérêts et des attentes des diverses parties prenantes. Cela suppose des mécanismes de gouvernance permettant aux diverses parties prenantes d'exprimer leurs attentes et d'être entendues des dirigeants et du conseil d'administration (B.Pigé, 2010).

\section{Une comparaison banques mutualistes et banques capitalistes}

L'étude et la comparaison entre les banques mutualistes et les banques capitalistes implique de s'intéresser aux différents modèles de coopératives existants et qui pourraient être susceptibles de trouver une place dans un secteur donné (ici le secteur bancaire). Elles agissent comme des entreprises capitalistes, sur de nombreux points. Contrairement aux idées reçues, ces coopératives sont des organisations compétitives dans la gestion de leurs capitaux : leur efficience est aussi grande que celle de leurs concurrentes privées et leurs fonctionnements aussi efficaces tout en n'étant pas centrés uniquement sur le profit. De plus, dans tous les cas, celles-ci cherchent toujours à réaliser un certain profit (ou surplus). Enfin, leurs spécificités se situent dans la recherche d'une utilité sociale : la satisfaction des besoins des membres du groupe d'appartenance (à travers la présence d'une même identité collective, d'une même origine sociale, d'une proximité idéologique) (A. Labye et al., 2002). On remarquera aussi que le système coopératif présente des règles spécifiques de fonctionnement, par opposition aux autres formes collectives d'organisation. L'homme est au centre du modèle (C.Vienney, 1994 ; J. Defourny, 1990 ; H. Desroches, 1976). Mais le fonctionnement coopératif allie logique privée de management (principes de gestion, aboutissant à la réalisation d'un résultat positif) et fonctionnement plus associatif (élection, votes...). 


\subsection{Des systèmes que tout semble opposer ...}

Ces deux systèmes semblent s'opposer sur leur origine et leur fonctionnement, l'organisation interne et enfin la rémunération des acteurs en présence.

\subsubsection{Origines et fonctionnements}

Ces deux types de banques ne sont pas construites sur les même modèles de développement. En France, la loi du 10 septembre 1947 structure la gouvernance coopérative sur quatre principes :

- la gestion démocratique ou participative de l'organisation ;

- la double qualité des membres ;

- la lucrativité limitée de la structure ;

- la solidarité.

De plus, pour développer et administrer les structures locales, cette forme d'organisation s'appuie aussi sur un autre aspect fondamentalement différent par rapport à une banque capitaliste : l'engagement bénévole des sociétaires qui sont élus en Assemblée Générale locale pour administrer la caisse. C'est leur qualité de sociétaire qui les rend potentiellement administrateurs. De même, aucune rémunération ne leur est attribuée, même si l'investissement humain peut y être très important. Ils peuvent simplement être défrayés de certaines dépenses engagées dans le cadre de leurs activités bénévoles, dans la limite maximale du SMIC.

Quant aux banques capitalistes, la cause de la création n'est bien évidemment pas la même. La prise en compte des besoins de leurs clients se réalise dans le but de réaliser un profit pour les actionnaires. Bien évidemment, la construction de la gouvernance de l'organisation est liée à la question de la majorité des votes en Assemblée Générale pour la désignation des membres du Conseil d'Administration et donc de ses dirigeants. Le bénéfice est distribué à travers le versement de dividendes aux actionnaires. 


\subsubsection{Organisation interne}

L'organisation interne de ces deux formes de banque est aussi différente. Elle passe par deux dimensions : l'organisation des pouvoirs entre le niveau local et le siège ainsi que la répartition des pouvoirs de direction.

Dans les structures mutualistes, l'échelon essentiel est la structure locale la plus proche du terrain. Il s'agit, par exemple, de la « caisse » locale au Crédit Mutuel. Chaque caisse est légalement indépendante et paye ses impôts selon ses résultats. Chaque caisse est également généraliste et cherche à répondre à l'ensemble des besoins de ses clients - sociétaires.

Les échelons régionaux (la caisse régionale) et nationaux (la fédération ou confédération) jouent un rôle de coordination et de conseil, en élaborant une stratégie commune ainsi que des produits ou en fournissant des moyens informatiques. Ces échelons sont des outils de représentation et d'administration, qui ne disposaient généralement, au départ, que de peu de pouvoirs et de poids politiques dans le fonctionnement de l'organisation : Ils sont au service des structures locales. On remarquera que le poids de ces structures régionales ou nationales semble s'accentuer (au détriment des structures locales).

Les pouvoirs de décision sont aussi clairement séparés. La dualité suivante est mise en œuvre : les salariés s'occupent de la gestion tandis que les administrateurs bénévoles prennent les décisions politiques. Les nominations du directeur et du président sont, en principe, indépendantes l'une de l'autre. Le directeur est nommé par la structure régionale, alors que le président est élu indirectement par les sociétaires via les administrateurs élus en Assemblée Générale.

A l'inverse, la banque capitaliste présente une structure centralisée où le siège impulse les décisions. La structure locale n'est qu'une agence de représentation et le niveau intermédiaire est structuré autour d'une dimension géographique en vue d'organiser au mieux les équipes de salariés. Il n'y a aucune structure juridique locale et le résultat ainsi que le paiement de l'impôt sont établis nationalement. Pour répondre aux besoins des clients, la société a développé des filiales spécialisées (assurances, crédit à la consommation, services informatiques...) qui diffusent leurs produits via le réseau et sont rémunérées pour cela. 
Le président du conseil d'administration est souvent également directeur général (il est donc P-DG)., bien que l'on puisse trouver aussi d'autres formes de gouvernance possibles : avec conseil de surveillance et directoire ou avec une séparation entre les fonctions de président et de directeur général.

\subsubsection{Rémunération des acteurs}

La question de la rémunération des différents acteurs fait, elle aussi, apparaître un écart important entre leurs habitudes respectives, que cela soit en matière d'apports, de répartition du résultat, de rémunération des salariés, des dirigeants ou des administrateurs.

Les parts sociales des mutualistes reçoivent un intérêt fixe indépendant du résultat de la société, alors que la détention d'actions des banques capitalistes est rémunérée par le versement de dividendes en fonction du résultat de l'entreprise.

Dans les banques mutualistes, il n'existe pas de commissions sur les ventes, les salariés sont considérés comme des «conseillers de clientèle ». Dans les banques capitalistes, la recherche du profit implique une incitation des commerciaux (appelés «chargés de clientèle ») à travers la mise en place de commissions sur objectifs.

En matière de rémunération des dirigeants, les banques mutualistes offrent des rémunérations plus faibles et moins incitatives que leurs concurrents privés. Baudoin Prot, directeur général de la BNP Paribas, a perçu en 2010 la somme de 2713 015€, dont $950000 €$ de salaire fixe et $1674053 €$ de variable ; Frédéric Oudéa, P-DG de la Société Générale, a perçu en 2010 la somme de $4350000 €$, dont $850000 €$ de salaire fixe et $3200000 €$ de variable. Par opposition, Michel Lucas, directeur général du Crédit Mutuel, a perçu pour la même année la somme de 1113 195€, dont $1100000 €$ de salaire fixe ${ }^{1}$. De même, pour la présidence du conseil d'administration, Michel Pébereau, président de BNP Paribas a perçu en 2010 la somme de $1411284 €$ dont $700000 €$ de fixe et $670000 €$ de variable, alors qu'Etienne Pflimlin, président du Crédit Mutuel, a perçu la somme de $630141 €$, dont $620000 €$ de fixe.

\footnotetext{
${ }^{1}$ Les Echos, 26 mai 2011.
} 
Enfin, les administrateurs mutualistes sont défrayés de leurs frais mais non rémunérés, même si l'investissement humain peut y être très important. Par contre, l'administrateur d'une banque capitaliste perçoit des jetons de présence. Ainsi, l'administrateur de BNP Paribas ${ }^{2}$ présent aux sept séances annuelles du conseil en 2010 a perçu $29728 €$ dont $14864 €$ (50 \%) au titre de la part fixe et $2123,43 €$ par séance de conseil où il était présent.

\subsection{Mais qui se structurent de façon semblable pour répondre à des contraintes identiques}

Les banques, malgré leurs statuts différents, obéissent à des contraintes identiques exigées des marchés financiers et de l'État. Pour pouvoir y répondre au mieux et dans des conditions acceptables pour le développement de leurs activités, elles sont alors contraintes de se développer de façon similaire pour atteindre la taille critique, ce qui aboutit aussi à des fonctionnements qui se ressemblent.

\subsubsection{Exigences des marchés financiers et de l'Etat}

En règle générale, les lois et normes qui s'appliquent au secteur bancaire ne font aucune différence entre les banques mutualistes et les banques capitalistes. Ainsi, la loi bancaire de 1984 a décloisonné les métiers bancaires, en ouvrant à la concurrence des domaines précédemment réservés aux mutualistes : milieux agricoles, ouvriers, PME. Les banques peuvent vendre tous les produits réglementés sans contraintes liées à leur structure juridique : il y a désormais généralisation du livret $\mathrm{A}$ et du livret Bleu, disparition des prêts bonifiés du Crédit Agricole pour les agriculteurs, etc.

De même, les normes IFRS, tout comme les normes Bâle II et Bâle III s'appliquent à toutes les banques. Les règles prudentielles sont identiques de même que les exigences de fonds propres. Même dans le domaine environnemental et social, les contraintes de la loi NRE, via les développements du Grenelle II de l'environnement, s'appliquent à compter de 2011.

\footnotetext{
${ }^{2}$ Rapport Annuel 2011
} 


\subsubsection{Atteindre la taille critique}

Une des réponses à ces multiples contraintes financières qui alourdissent le fonctionnement et limitent les actions de l'ensemble des banques, est la recherche de la taille critique. Celle-ci passe par le rachat de réseaux concurrents en France ou à l'étranger, la réponse à des besoins spécifiques, la standardisation des produits et le regroupement des activités de back-office, et le développement de sociétés de capitaux sous forme de filiales pour les banques mutualistes.

Les opérations de rachat de réseaux mêlent indistinctement banques capitalistes et banques mutualistes : rachat du Crédit du Nord par la Société Générale, du CIC par le Crédit Mutuel, de LCL par le Crédit Agricole, de la banque San Paolo par le Crédit Agricole, de la banque Palatine par la banque Postale, de Citibank par le Crédit Mutuel, de Fortis et BNL par BNP Paribas, d'Emporiki par le Crédit Agricole, etc.

Chaque réseau bancaire développe parallèlement des structures spécifiques. Par exemple, pour le Crédit Mutuel, le CME (Crédit Mutuel Enseignant), le CMPS (pour les professionnels de la santé), une structure de «Banque Privée », le CMI (financement et activités autour de l'immobilier: gestion, intermédiaire...), la BECM (pour les grosses entreprises), Cofidis (le crédit à la consommation, entreprise rachetée et non créée de toute pièce), etc. La structuration est parfois légèrement différente (ainsi que les dénominations des différents services ou domaines), mais on la retrouve partout d'une façon plus ou moins poussée et plus ou moins formelle.

On assiste aussi à une standardisation des services, que ce soit vis-à-vis des clients (bancassurance, assurances de complémentaires santé, services de sécurité, accès internet, offres téléphoniques, placements éthiques ou solidaires pour soutenir certaines associations humanitaires ou fondations, etc.) ou en interne. Les banques aménagent la partie invisible pour le client pour mutualiser des coûts : ainsi la Société Générale et le Crédit du Nord procèdent-ils actuellement à une refonte de leurs systèmes informatiques pour arriver à un élément commun, l'assurance ou la gestion d'actifs pour LCL et le Crédit Agricole sont regroupés respectivement dans Pacifica et Amundi (qui est aussi le fruit d'un rapprochement 
avec la gestion d'actifs de la Société Générale...). C'est par ce moyen-là, que les différentes fédérations ou caisses régionales du Crédit Mutuel, du Crédit Agricole, des Banques Populaires, chacune pour son réseau, sont amenées à se rapprocher et peu à peu à fusionner.

Enfin, pour disposer d'un accès à la bourse et à ses sources de financement, les différents réseaux coopératifs, malgré un positionnement mutualiste, ont maintenu voire même peu à peu renforcé leurs structures cotées: bien que détenue à 98,7 \% par le Crédit Mutuel, le CIC reste toujours coté ; de son côté, le Crédit Agricole a placé en bourse $30 \%$ du capital de la structure CASA (Crédit Agricole SA) qui via la SAS la Boetie détient $25 \%$ du capital des caisses régionales et fait ainsi remonter à ses actionnaires des dividendes; enfin, Natexis, malgré ses déconvenues et ses énormes pertes, reste toujours cotée par la BPCE.

\subsubsection{Fonctionnement interne similaire}

La rationalisation de la relation client et l'augmentation de la taille des portefeuilles clients gérés par les salariés favorisent l'uniformisation: chez les mutualistes, les « conseillers » ont été remplacés par des « chargés de clientèle ». La préférence pour les liens commerciaux est mise en valeur (avec la recherche de l'utilité marchande et de la minimisation des risques et des coûts du client). Bien que les publicités du Crédit Mutuel en novembre 2010 annoncent fièrement que les chargés de clientèle ne sont pas rémunérés à la commission, ce point a été proposé au Crédit Mutuel Arkéa (Bretagne) et a suscité de vives réactions en interne.

Le contrôle hiérarchique est renforcé (fixation d'objectifs, primes pour atteinte de ceuxci) et le management devient similaire pour les établissements privés et les caisses mutualistes. Parfois, les même dirigeants se retrouvent dans deux formes d'organisation : par exemple au CIC (banque capitaliste filiale) et au Crédit Mutuel (banque mutualiste société mère) où l'équipe dirigeante autour de $\mathrm{M}$. Lucas est la même. 


\section{Le localisme et la proportionnalité, principes fondateurs du mutualisme? L'étude de la FNIM.}

"L'on connaît beaucoup mieux les besoins de sa ville que ceux des autres villes; et on juge mieux de la capacité de ses voisins que de celle de ses autres compatriotes. Il ne faut donc pas que les membres du corps législatif soient tirés en général du corps de la nation; mais il convient que, dans chaque lieu principal, les habitants se choisissent un représentant $»^{3}$

L’Assemblée générale de la Fédération Nationale Interprofessionnelle des Mutuelles (FNIM) du 6 décembre 1990 prévoit :

- un accès direct des «mutuelles de base » à la Fédération; c'est le «localisme » fondamental fondé sur l'idée de la «subsidiarité » qui légitime le fait de la décision politique, adaptée à un deuxième degré selon les critères de Frédéric.-Guillaume. Raiffeisen (Ph. Naszályi 2010),

- une représentativité proportionnelle par collège, aussi bien aux Assemblées générales qu'aux Conseils d'administration, qui sous-tend la présence la plus grande et le « consensus » que nous appellerons de large et profonde conviction dans le cadre d'un débat de qualité: "une entente rationnellement motivée », selon l'expression de Jürgen Habermas (1997).

Avec vingt-deux Assemblées générales de 1991 à 2005 et neuf modifications importantes de ses statuts, la FNIM est exemplaire de cette mise en pratique d'une représentativité de ses adhérents, tout comme de la volonté de les admettre en son conseil, parce qu'ils la rejoignent, et parfois aussi, pour combler les places de ceux qui la quittent, dans une parfaite application d'ailleurs de «la libre adhésion», valeur reconnue de l'économie sociale.

\footnotetext{
3 Montesquieu, De l'Esprit des Lois, in Oeuvres complètes, Paris 1838, 770 pages, page 266,
} 


\subsection{La Gouvernance par les mutuelles de base}

L'assemblée générale de la FNIM du 3 octobre 1992 illustre cette volonté de faire participer à la gouvernance de la Fédération tout membre de celle-ci. ${ }^{4} \mathrm{Ce}$ souci, qui n'est pas que formel, a amené à la création dans les statuts d'une «Commission administrative et des statuts » qui, à chaque assemblée générale, délibère sur l'application «de la règle mathématique $»^{5}$ et sur son adaptation stricte aux règles statutaires de représentativité pour calculer les délégués. L'assemblée générale du 17 juin 1994, ratifie l'augmentation du nombre d'administrateurs de 20 à 22 (le maximum statutaire était de 30 ) pour le collège des salariés (collège B) «du fait que plusieurs mutuelles ont rejoint notre Fédération $»^{6}$.

L'application de ce système complexe amène inévitablement des conflits ou des difficultés entre grandes et petites mutuelles. "J'entends dire ici et là que l'égalité de traitement de toutes les structures ne serait pas forcément respectée en toutes circonstances par la FNIM » ${ }^{7}$ s'exclame le président avant de fustiger de «telles mesures », propagées par des «égarés» qui font preuve d'une «incompréhension manifeste et totale des mécanismes démocratiques qui ont toujours prévalu à la FNIM». Il faut rappeler en effet, que c'est pour lutter contre "l'hégémonie» des grandes mutuelles de fonctionnaires, que des petites mutuelles ont quitté la FNMF (Fédération Nationale Mutuelle des Fonctionnaires, la fédération historique). La FNIM entend donc favoriser l'accueil des petites mutuelles; le nombre d'adhérents par mutuelle compte moins que l'application correcte des règles de proportionnalité. En effet, dès la création de la FNIM, des dispositions ont été prises afin que toute mutuelle, et ce quel que soit son effectif, soit représentée à l'Assemblée générale de la Fédération, grâce à l'article 15 des statuts.

${ }^{4}$ Le président indique qu'en fonction des dernières adhésions à la FNIM, la nouvelle composition du conseil, pour laquelle on constate deux postes vacants et un poste mis à disposition par l'UITSEM, permet de procéder à trois cooptations qui, bien entendu, seront réexaminées lors du renouvellement du conseil en 1993, qui tiendra compte des adhésions à la Fédération intervenue d'ici à cette date. PV de l'AG du 3 octobre 1992, page 6. FNIM.

${ }^{5} \mathrm{PV}$ de l'AG du 8 octobre 1993, page 5. FNIM

${ }^{6} \mathrm{PV}$ de l'AG du 17 juin 1994, page 6. FNIM

${ }^{7}$ Michel Hermant, discours de l'AG du 13 juin 1997, page 14. FNIM 
Aucun débat conflictuel grave n'a été relevé avant 2005 car les « grandes » mutuelles qui ont choisi la FNIM, acceptent, naturellement, "d'écrêter leur puissance et leur représentation $»^{8}$ au profit d'autres ayant moins de place. Ainsi pour l'Assemblée générale de 1991, des membres fondateurs $\left(\mathrm{UNIM}^{9}\right.$ et $\left.\mathrm{UITSEM}^{10}\right)$ décident de façon transitoire de n'avoir que 4 délégués au lieu de 12 dans le collège $\mathrm{G}$ «afin de ne pas déséquilibrer l'Assemblée générale ${ }^{11}$ illustrant la formule du président fédéral : «Le pouvoir, c'est l'envie et non la taille. Tout le monde doit pouvoir s'exprimer librement, quelle que soit sa taille, un groupe étant égal à une voix $\gg .{ }^{12}$ La démocratie directe est à ce prix, et la FNIM l'assume !

\subsubsection{La représentativité interprofessionnelle.}

L'interprofessionnalité est un autre des éléments fondateurs de la FNIM. C'est pour lutter contre la mainmise des mutuelles de fonctionnaires sur la FNMF que Michel Hermant a engagé, avec d'autres personnalités, le processus de création. "Bien sûr, les fonctionnaires sont numériquement nombreux chez nous, y compris aux postes de responsabilité. Mais ils sont élus! Est-ce ma faute si les fonctionnaires sont ceux qui peuvent le plus facilement se dégager de leurs obligations professionnelles? ...» ${ }^{13}$ reconnaissait alors, René Teulade, président de la FNMF et en même temps, de la Mutuelle de Retraite de la Fonction publique !

L'article 15 des statuts de la FNIM est l'expression de ce principe de représentation de la « nouvelle Mutualité » avec cinq collèges :

- les jeunes, les étudiants, collège $\mathrm{A}$,

- les salariés qui occupent le collège $\mathrm{B}$ et qui ont été distingués des fonctionnaires et titulaires,

- les professions non salariées, qui forment le collège $\mathrm{C}$,

- les professions agricoles et les retraités forment les collèges $\mathrm{E}$ et $\mathrm{F}$,

\footnotetext{
${ }^{8}$ Michel Hermant, PV du Conseil d'administration du 22 juin 2001, page 12. FNIM

${ }^{9}$ Union Nationale des Informateurs Médicaux, Nord Pas de Calais

${ }^{10}$ Union de mutuelles étudiantes UITSEM

${ }^{11}$ PV du Conseil d'administration du 22 juin 1991. FNIM

${ }^{12}$ Michel Hermant, PV du Conseil d'administration du 22 juin 2001, page 12. FNIM

13 Controverse autour de l'influence des fonctionnaires. Le Monde, 2 mars 1990, article de
} Jean-Michel Normand. 
- le dernier collège enfin $(\mathrm{G})$ concerne les deux unions fondatrices : l'UNIM et l'UITSEM,

Au moment de la réforme des statuts de 2002, Michel Hermant rappelle clairement que « la FNIM avait opté pour cette structure pour ne pas reproduire le système qui faisait que des fonctionnaires de la Fédération "d'en face », sans être majoritaires et sans que cela soit rarement leur argent, étaient, par le biais, entre autres, d'unions, aux commandes de l'ensemble du dispositif $\gg{ }^{14}$.

Les choses ne se passent pas tout à fait selon les « alchimies complexes ${ }^{15}$ du président. Avec la réforme de 2002, rendue obligatoire par la réforme du Code de la mutualité, il ne reste plus dorénavant que deux collèges : «les membres fondateurs» qui sont toujours l'UNIM et l'UITSEM et « les membres cotisants », c'est-à-dire les cinq collèges qui figuraient jusque-là ${ }^{16}$

Cette représentativité institutionnelle sans cesse réaffirmée, s’illustre par la volonté de faire participer tous les entrants à la gouvernance de la FNIM, aussitôt que possible et sans attendre les échéances des mandats. Ainsi, à la question de la participation de certains organismes nouvellement adhérents, au Conseil d'administration, le président, suivi par ce dernier, précise que «rien ne s'oppose à ce qu'un de leurs représentants soit coopté par le Conseil, dès lors que leur adhésion effective aura été entérinée par l'Assemblée générale »17.

Cette position qui demeure pratiquée durant toute la période amène une relative instabilité de la composition des Conseils et un risque d'absence de cohérence politique, comme le souligne le délégué général en 2005. "J'ai sous les yeux la liste des administrateurs renouvelables en juin 2005 ; c'est une valse permanente de démissions et de cooptations $»^{18}$.

${ }^{14}$ Réponse de Michel Hermant, à Colette Fuchs (Muta santé) PV du Conseil d'administration du 16 janvier 2002, page 7. FNIM

${ }^{15}$ C'est d'ailleurs cette savante alchimie de représentation interprofessionnelle-inspirée par la FNMFpermettant à tous d'être représentés que le Code de la mutualité, imposé par Martine Aubry, a broyée.

${ }^{16} \mathrm{PV}$ de 1 'AG du 27 mars 2002, page 3. FNIM

${ }^{17} \mathrm{PV}$ du Conseil d'administration du 29 mai 1992, page 7. FNIM

${ }^{18}$ Gilles Marchandon, délégué général de la FINM de 1999 à 2006. PV du Conseil d'administration du 11 mai 2005, page 10. FNIM 
Une illustration s'il en était besoin, que la pratique démocratique ne coïncide pas forcément avec une efficience de gouvernance, du moins selon les critères institutionnalisés. Mais au fond, la FNIM ne prétend pas y répondre et, comme on le verra plus clairement par la suite, manque de disparaître pour avoir « copié » un fonctionnement plus orthodoxe.

\subsubsection{La proportionnalité des délégués ou les limites à « un homme une voix »}

Les sociétés coopératives, les mutuelles ou les associations qui composent l'économie sociale sont des organisations où s'applique le principe de vote « un homme = une voix ». La libre adhésion et la libre sortie des membres ou sociétaires en sont les compléments. C'est dans cet esprit, mais avec des nuances que la FNIM organise la représentation de ses adhérents.

«Le nombre des délégués élus par chaque groupement mutualiste est proportionnel à l'effectif de chacun d'eux dans chacun des collèges. ». Cette proportionnalité est fonction du nombre de chefs de famille déclaré au 31 décembre de l'année précédente ou au moment du paiement des cotisations. Toutefois, cette proportionnalité est réglementée pour éviter les abus de domination par un groupe. En effet, si chaque groupement adhérent doit être représenté par un délégué au moins, le maximum prévu pour les grands groupements permet d'empêcher une mainmise ${ }^{19}$.

Par ailleurs, l'article 28 des statuts interdit à un organisme de la FNIM de disposer de la majorité des sièges du Conseil d'administration, ce qui vise à éliminer tout risque de «gouvernement tyrannique» au profit d'une seule catégorie de membres comme la FNIM accusait la FNMF de le pratiquer.

Même lorsque les statuts auront été réformés après le changement du Code de la mutualité de 2001, des garde-fous empêchent une seule mutuelle d'être représentée au Conseil par plus du quart des administrateurs. On peut illustrer la stricte application de ce principe,

${ }^{19}$ Dans les faits, si un groupement adhérent obtenait un nombre de délégués, au titre de la représentation proportionnelle, supérieur au tiers moins deux du nombre total de délégués à l'Assemblée générale, sa représentation à ce titre serait ramenée au maximum autorisé et le nombre de délégués en excédent, réparti au prorata dans chaque collège dans lequel ce groupement est présent, sera attribué aux autres groupements. 
lorsque PREVIADE, qui est le plus gros organisme adhérant à la FNIM, par le biais du mécanisme de la représentation « maxima », se voit amputé, non sans protestations d'ailleurs, de 2 délégués à l'assemblée (26 au lieu de 28$)^{20}$.

Cette représentation au plus près des mutuelles de base qui a généré la modification des statuts de 1991, 1992 et 1998, n'est pas sans inconvénient. Michel Hermant, pourtant à l'origine de ce processus y voit, en 1999 une «mentalité de tribu gauloise », avec "des courants, sous-groupes, fractions voire mutuelles composant individuellement la FNIM ${ }^{21}$ ? On est loin de la stabilité d'ordinaire requise dans une gouvernance de démocratie représentative classique. La FNIM se situe «dans la puissance subversive toujours neuve et toujours menacée de l'idée démocratique » (Jacques Rancière, 2005).

\subsection{L'effectivité de la prise de décision par des élus ou la démocratie participative}

Plus qu'une forme d'instabilité, c'est par deux points majeurs de «déviance démocratique » que nous souhaitons démontrer ce qu'est l'idéal-type démocratique, tel que la FNIM le revendique comme sa marque originelle. Le pouvoir donné à des comités ad hoc, au détriment des instances statutaires et la dyarchie (président/délégué général), sont les éléments d'une sorte de "revers évidé » de la démocratie qui vont apparaître comme une maladie de la FNIM, au tournant de l'an 2000, pour disparaître cinq plus tard, suite à la réaction du «corps sain » expurgeant l'intrus. Entre temps, la petite fédération aura failli sombrer ou être absorbée.

La FNIM est une fédération nationale et non une association ou une coopérative locales, ce qui nous prive de tout référentiel comparatif mesurable. Cela sous-entend que les élus ou les représentants, en général non parisiens, depuis l'origine de la FNIM, doivent se réunir à Paris ou dans une des villes choisies pour l'organisation tournante de l'Assemblée générale. A partir de 2003, les réunions et les assemblées ne se tiennent plus qu'à Paris. Les «élus »

\footnotetext{
${ }^{20} \mathrm{PV}$ du Conseil d'administration du 11 avril 1997, page 12. FNIM

${ }^{21}$ Discours de l'AG du 11 juin 1998, page 6. FNIM
} 
viennent au délégué général qui ne prend pas le temps de se déplacer en Province. Ce n'est pas une singularité puisqu'une structure bancaire coopérative comme la caisse d'épargne en Ile-de-France fonctionne sur ce même système.

L'analyse de l'ensemble des 154 réunions qui ont été répertoriées de 1990 à 2005, a permis de relever une tendance constante et linéaire d'augmentation de l'absentéisme aux réunions. La présence aux assemblées générales est plus importante qu'aux réunions parisiennes des conseils, où l'absentéisme des élus a fréquemment entraîné, à partir de 2000, une impossibilité de délibérer faute de quorum. Le bénévolat de mutualistes répartis dans toute la France peut en être une des explications, sans qu'il soit suffisant à justifier seul cette désaffection. "Neuf heures de train pour 2 heures de réunion! Réunions inutiles, tout semblant joué dans le travail fait probablement au Bureau $»^{22}$ en dit long sur la perception du pouvoir réel, en 2005 .

La « nouvelle gouvernance de la FNIM» avec sa dyarchie est bien, pendant un court temps (2000-2005 dans sa plus grande durée), une pratique où des commissions ad hoc remplacent les organes statutaires et où, des non-élus sont missionnés pour agir à la place des élus. Le Code de la mutualité, dans son dernier avatar de 2001, en instituant le duo « président et directeur général » et reléguant au second rôle, les conseils élus, a accentué cette prise en main des organismes théoriquement démocratiques de l'ESS par une technostructure qui a, pour elle, l'avantage de la permanence sinon de la compétence technique. La FNIM semble en avoir mesuré le danger et avoir réussi à l'écarter en 2006.

Les propos de Paul Collomb, pourtant chef de brigade de l'Autorité de contrôle des assurances et des mutuelles (ACAM), en rappelant en 2009 le rôle premier de l'élu et le poids politique qu'il doit tenir, confirment que, comme dans son combat pour la représentativité, la FNIM peut constituer un modèle de gouvernance privilégiant le «politique » sur le « technique »: «La question n'est pas de présenter une liste de diplômes. Un bon

\footnotetext{
22 Jacques Gontiers (SMT), PV du Conseil d'administration du 11 mai 2005, page 8. FNIM.
} 
administrateur est celui qui connaît sa responsabilité et ses devoirs pour guider au mieux sa mutuelle. $»^{23}$.

\subsubsection{Des Comités ad hoc plus que des Commissions statutaires.}

La refonte des statuts en 2002, amène la création d'une commission « ad hoc » qui rend caduque la Commission des statuts, «pourtant statutaire » et existant depuis l'origine. Il paraît donc à la fois paradoxal, mais aussi dans la logique évoquée plus haut de «tribus gauloises », d'entendre le président et fondateur Michel Hermant déclarer "qu'elle ne correspond aujourd'hui à plus rien, tout le monde ayant voulu y avoir un représentant ».

La préparation de l'Assemblée générale du 28 juin 2002 amène son successeur, suivi par le Bureau ${ }^{24}$, à proposer la création d'un groupe de travail. Or, la Commission administrative et des statuts est la seule commission dont les membres sont désignés directement par l'Assemblée générale. Elle incarne la démocratie directe. De plus, elle est dotée du pouvoir de régler les conflits qui surgissent, notamment en Assemblée générale (article 15). Lui retirer son pouvoir est à l'évidence un choix qui s'inscrit en contradiction majeure avec les principes fondateurs de la FNIM.

En 2004, lorsque l'un des administrateurs propose d'élargir la réflexion de développement à la fonction publique en entier, alors que le délégué général n'a abordé que la fonction publique territoriale, le président préfère clore le débat, en déclarant qu'il «ne veut pas, dans la mesure où un groupe de travail est constitué, faire perdre du temps au Conseil en refaisant deux fois la même chose $»^{25}$. Discuter hors du cadre est devenu "perte de temps », comment trouver meilleure illustration de l'abandon de la démocratie participative ? C'est aussi un groupe de travail de quatre personnes ${ }^{26}$ qui est chargé de préparer ou non un séminaire de réflexion de deux jours sur l'avenir de la FNIM et le président de ponctuer : «le Conseil sera informé du résultat ».

\footnotetext{
${ }^{23}$ La Gouvernance des mutuelles, Plénières, Décid'assur, 25 mars 2009, page 3.

${ }^{24} \mathrm{Il}$ est à noter que nous ne disposons plus aux archives d'aucun compte rendu de Bureau depuis 2000

${ }^{25} \mathrm{PV}$ du Conseil d'administration du 25 février 2004, page 8. FNIM

${ }^{26}$ PV du Conseil d'administration du 8 décembre 2004, page 3 et 4 . FNIM
} 


\subsubsection{La dyarchie}

La « dyarchie » découle directement de ces pratiques. Durant cette période, la FNIM n'a pas échappé à la tentation rencontrée un peu partout dans le monde mutualiste, parfois depuis longtemps, de considérer que la technicité des questions traitées fait de « l'Élu », nonspécialiste par définition, une quantité secondaire dans la décision. Cette pratique est attestée dans les firmes capitalistes où "le niveau élevé de complexité des sociétés implique normalement, que le Conseil d'administration se décharge lui-même de l'essentiel des fonctions de décision et de contrôle sur les agents internes de la société, (pour ne garder) que le contrôle ultime de leurs décisions » (Gérard Charreaux et Jean-Pierre Pitol-Belin, 1985). La technostructure, qui vide de sens toute démocratie politique, est un danger que pointent tous les partisans de l'économie sociale. «La complexité des problèmes gestionnaires, surtout dans les grandes organisations mutualistes, tend à éloigner les sociétaires des lieux véritables où se prennent les décisions. La tentation existe d'assimiler la règle démocratique à une contrainte, contrariant la réactivité nécessaire des groupements face aux mutations incessantes de l'économie et de la société.» (Patrick Gianfaldoni et Nadine Richez-Battesti, 2008). Ce sont des arguments similaires qui sont utilisés ce 8 décembre 1999, à la FNIM par cette volonté affirmée de donner «un fonctionnement moderne, un fonctionnement efficace qui ne posent pas de problème et conviennent au maximum d'entre nous $»^{27}$. L'enjeu à la FNIM est de cette nature. Les causes extérieures : code de la mutualité, réforme de la CNAMTS... ou de manière moins précise et plus grandiloquente : "l'aube du nouveau millénaire » ne sont que des prétextes. Ces éléments obligeraient «à tourner une page nouvelle du grand livre de la Fédération», car «les choses ne seront plus jamais comme elles étaient hier en ce qui concerne la Mutualité $\gg{ }^{28}$.

Dans cette logique, le cadre supérieur qui doit être recruté doit disposer de « compétences pointues ${ }^{29}$ et être un «expert de haut niveau (si possible, grandes écoles

\footnotetext{
${ }^{27}$ Michel Hermant, PV de l'AG du 8 octobre 1999, Page 2. FNIM

${ }^{28}$ Michel Hermant, discours de l'AG du 8 décembre 1999, page 3. FNIM

${ }^{29}$ PV du Conseil d'administration de la FNIM du 13 février 1998, page 4. FNIM
} 
plus expérience : ENA, HEC, ESSEC) » ${ }^{30}$. Ce «chargé d'études » devient finalement un «délégué général» ce qui à l'évidence ne recouvre plus la même réalité de pouvoir. La discussion d'alors montre s'il en était besoin toute l'ambiguïté du problème posé à la démocratie mutualiste, en décrivant la «difficulté de séparer partie politique et partie technique » pour que «les rôles de chacun ne s'inversent pas ${ }^{31}{ }^{1}$ et afin que «le délégué général (ne) puisse supplanter le président $»^{32}$.

La FNIM, si elle entre dans une nouvelle définition de ses pouvoirs décisionnaires en 2000, s'y engage davantage du fait des circonstances et des rencontres d'hommes, que d'une volonté délibérée et organisée. Le phénomène n'a donc pas pris l'ampleur constatée dans d'autres structures comme les banques coopératives où, "non seulement la démocratie représentative (à travers les Assemblées générales pour l'essentiel) est encadrée par des mécanismes formels maîtrisés et contrôlés par un " haut encadrement », constitué par des administrateurs élus et épaulés par une technostructure efficace, mais de plus, la démocratie participative n'a que peu de consistance sur les questions stratégiques dans les conseils locaux ou régionaux. » (Patrick Gianfaldoni et Nadine Richez-Battesti, 2008). En effet, toute la démocratie de la FNIM n’a pas été «gangrenée » par cette arrivée au pouvoir de la technostructure, sans doute par la courte durée de l'expérience dyarchique et par la réaction des élus dont nous avons constaté l'engagement militant de conviction.

Plus que les assemblées générales de la FNIM, qui demeurent vivantes voire vives, mais qui sont, en fait, ouvertes à des mutualistes militants et non à des sociétaires, ce sont les Conseils d'administration qui semblent avoir souffert, pendant cette période, d'une appropriation du pouvoir de l'Élu par les non-élus. Toutefois, lors de l'Assemblée générale de 2001 et pour la première fois, le délégué général «seconde » le secrétaire fédéral comme secrétaire de séance. Sans être un élément déterminant, cette évolution l'amène ${ }^{33}$ à rédiger les

\footnotetext{
${ }^{30}$ Intervention de Philippe Delemarre, PV du Conseil d'administration du 28 mai 1998; page 3. FNIM

${ }^{31} \mathrm{PV}$ du Conseil d'administration du 21 novembre 1998, page 2. FNIM

${ }^{32} \mathrm{PV}$ du Conseil d'administration du 21 novembre 1998, page 7. FNIM

33 "Le secrétaire (Jean-Paul Balâtre) délègue à M. Gilles Marchandon, en sa qualité de délégué général de la FNIM, ses pouvoirs en matière de convocation, de rédaction des procès-verbaux, de conservation des archives et de tenue $d u$ fichier des adhérents .M. Marchandon agira sous la responsabilité du secrétaire, à
} 
procès-verbaux, voire à les signer depuis $1999^{34}$. C'est le délégué général qui, à la demande du président, traite de la proposition de la FNIM en matière de « nouvelle gouvernance » à l'Assemblée générale de Lyon de $2003^{35}$, et présente la modification des statuts ${ }^{36}$.

La déperdition du rôle du Bureau est également très sensible. Pourtant organe statutaire, c'est lui qui avait initialement autorité ${ }^{37}$ sur le délégué général. Il se voit retirer ce pouvoir au profit du Conseil d'administration dont on vient de constater qu'il est privé de débats. Il laisse apparaître le vrai enjeu de la dyarchie : président/délégué, avec les risques soulevés plus haut. À partir de 2000, on ne retrouve plus guère de procès-verbaux du Bureau. ${ }^{38}$ Cela ne veut pas dire que le Bureau ne se réunisse plus, puisqu'il y est fait mention de temps à autre, cela signifie que sans procès-verbaux et donc sans transparence, il n'est plus un élément moteur de décision de la Fédération dont le pouvoir repose désormais sur le président et le délégué général. De plus si «le Bureau est, en ce qui le concerne, l'instance chargée de traiter les affaires courantes ${ }^{39}$, comme sa mission en est définie, il n'a effectivement plus aucune raison de fonctionner puisque les affaires courantes sont résolues par le délégué général et son équipe, avec l'accord (téléphonique) du président fédéral.

Il ne reste plus alors qu'à marginaliser le président. Sans tenir compte de l'importance des sujets, nous avons relevé simplement le nombre de fois où le président et le délégué général prenaient la parole au cours de conseils d'administration de la FNIM, en l'occurrence,

charge pour lui de lui rendre compte. Le secrétaire l'autorise également à subdéléguer la partie des pouvoirs qu'il jugera utile au bon fonctionnement administratif de la Fédération. » PV du Conseil d'administration du 24 juin 2005, page 4. FNIM.

${ }^{34}$ « il paraît indispensable (au président) que le (délégué général) puisse bénéficier des délégations nécessaires...et il souhaiterait que le secrétaire délègue ses pouvoirs (au délégué général)» PV du Conseil d'administration du 11 mai 1999, page 3. FNIM.

${ }^{35} \mathrm{PV}$ de l'AG de la FNIM du 20 juin 2003, page 2. FNIM

${ }^{36} \mathrm{PV}$ de l'AG de la FNIM du 20 juin 2003, page 4. FNIM

${ }^{37}$ Conseil d'administration du 21 novembre 1998

38 2000, un PV de réunion de Bureau,

2001, aucun PV

2002, aucun PV

2003, aucun PV

2004, aucun $P V$

2005, un seul PV officieux qui concerne la crise qui éclate pour la fixation des délégués pour la mutuelle du président.

${ }^{39}$ Rapport d'orientation de Michel Hermant, annexe au PVde l'AGde la FNIM du 5 juin 1998, page 4. FNIM 
le premier de l'année, pendant la période que nous avons constatée être symptomatique en matière de déviance.

Tableau 1 : Nombre d'interventions au cours des Conseils d'administration de 2001 à $2004^{40}$

\section{Nombre d'interventions au cours du Conseil d'administration}

Dates des Conseils

31 janvier 2001

16 janvier 2002

15 janvier 2003

14 janvier 2004 délégué général

$$
2
$$

26

29

23 président

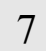

41

6

9

Les données brutes illustrent l'inflexion prise à partir du changement de président, lequel n'intervient que très peu et sur aucune des questions importantes mises en discussion.

Cette dyarchie, qui amène le délégué général à prendre de plus en plus le pas sur le président, provoque quelques remous qui s'expriment davantage à la sortie du mandat du successeur du Président-fondateur. Tel président de Mutuelle «s'étonne qu'un délégué général puisse représenter la Fédération en justice » ${ }^{41}$ et que Combat mutualiste, le journal de la FNIM que dirige ce même délégué général, puisse annoncer des faits avant que les textes soient connus des administrateurs. Ce qui, dit-il, «l'a scandalisé ».

Sans y faire attention, mais les lapsi sont souvent révélateurs, le secrétaire fédéral, lors du Rapport qu'il établit pour l'Assemblée générale de juin 2005, a cette formule : «je voudrais également, avant de clore ce rapport, adresser mes vifs remerciements et félicitations aux collaborateurs de la FNIM, dirigée par le (délégué général) ${ }^{42}$.

\section{Conclusion: Le modèle mutualiste est-il un modèle de gouvernance démocratique?}

Les coopératives constituent une forme ancienne d'Organisation dont l'heure de gloire semble avoir coïncidé avec la crise du capitalisme des années 1930. La crise économique et

\footnotetext{
${ }^{40}$ Source : Naszályi Ph (2011) La Nouvelle Mutualité ", utopie ou refondation

${ }^{41} \mathrm{PV}$ de l'AG du 24 juin 2005, page 6. FNIM.

${ }^{42}$ Rapport du secrétaire à l'Assemblée générale de la FNIM du 24 juin 2005, page 7. FNIM.
} 
financière que nous traversons encore si elle a rappelé certaines caractéristiques de la crise de 1929 n'en a heureusement pas eu les conséquences. Elle a néanmoins soulevé à nouveau la question de la finalité des Organisations dans la vie économique et sociale de nos Sociétés. L'approche théorique des organisations permet désormais, avec de nouveaux outils, de mieux comprendre les spécificités et l'avantage comparatif réel des coopératives dans le monde économique contemporain.

L'hypothèse principale que nous posons est que l'enjeu principal des coopératives est de devenir les Organisations de référence dans l'application du modèle des parties prenantes tout en étant viables économiquement, ce qui suppose de résoudre les trois points suivants :

- Assurer une gestion efficiente des ressources qui permette de réaliser des transactions à un coût inférieur à celui qui aurait lieu sur le marché.

- Mettre en œuvre des mécanismes de gouvernance permettant l'expression et la prise en compte des intérêts des diverses parties prenantes.

- Mettre en œuvre des mécanismes de gouvernance permettant d'évaluer ex post l'action des dirigeants et la réponse apportée aux attentes des parties prenantes.

Il y a bien une différence de gouvernance entre une fédération de mutuelles et une entreprise capitaliste, même dotée de la meilleure des chartes éthiques et animée de l'esprit de la Responsabilité Sociale des Entreprises. Il existe également bien des différences entre deux entreprises mutualistes ou coopératives. L'étude de la FNIM a permis de souligner la contradiction organisationnelle des structures de l'économie sociale et solidaire qui, en se présentant comme une alternative à l'entreprise capitaliste, succombent à une autre forme d'appropriation illégitime du pouvoir, en privant les "propriétaires réels », que sont les sociétaires, de la démocratie. "Le système démocratique tel qu'il est pratiqué dans les mutuelles favorise l'autocontrôle et l'émergence de personnes issues de la base de l'organisation » estime Jean-Luc de Boissieu, secrétaire général du GEMA ${ }^{43}$, sans masquer

\footnotetext{
${ }^{43}$ Intervention de Jean-Luc de BOISSIEU, secrétaire général du Groupement des entreprises mutuelles d'assurances (GEMA), lors de la conférence de l'observatoire de la gouvernance du CJDES du 28 février 2008, page 21 .
} 
les incertitudes quant à la compétence qui sera exigée de ces administrateurs dans le cadre de l'évolution des règles européennes de solvabilité.

Faire reposer sur « la représentativité des composantes » et "l'effectivité de la prise de décisions par des élus ", la nouvelle gouvernance des entreprises coopératives ou mutualistes est l'élément qui définit idéalement une organisation de l'économie sociale par rapport à une société de capitaux, même en cours de démocratisation.

Cet idéal-type de démocratie de gouvernance fait appel à la fois à la démocratie représentative, qui ne peut fonctionner qu'avec une juste répartition des mandataires et leur assiduité aux élections, et à la démocratie participative à l'échelon des élus, où conseils et bureaux doivent définir les politiques par un consensus.

Parce que la décision doit se prendre dans un «monde incertain» et «parce les avancées des sciences et techniques ne sont plus contrôlables » (Michel Callon et al., 2001), ou ne semblent plus l'être, la FNIM a tenté de corriger la démocratie directe qui est sienne par le recours à l'expert au sens que prédisait le comte de Saint-Simon «L'administration des choses remplacera le gouvernement des hommes ", véritable préfiguration de la technostructure de James Burnham (1941) ou de John Kenneth Galbraith (1967). Dans l'esprit pragmatique de notre étude, nous ne pouvons que souscrire à l'idée que développe John Dewey qui constate que «c'est la personne qui porte la chaussure qui sait le mieux si elle fait mal et où elle fait mal, même si le cordonnier est l'expert qui est le meilleur juge pour savoir comment y remédier. [...] Une classe d'experts est inévitablement si éloignée de l'intérêt commun qu'elle devient nécessairement une classe avec des intérêts particuliers et un savoir privé - ce qui, sur des matières qui concernent la société, revient à un non-savoir» (John Dewey, 2003).

La FNIM s'est trompée dans cette démarche du recours à l'expert, parce que les solutions apportées n'entraient pas dans la logique qui avait présidée à sa création. Il semblait apporter des réponses techniques, fruits de son idéologie, mais au fond inadaptées, voire destructrices. Cela, comme nous l'avons vu, a permis de ciseler un modèle de gouvernance, 
fondé sur le conseil élu et la prise de décision effective par ce dernier, comme critères fondateurs.

Passer à un modèle de parties prenantes suppose que l'entreprise coopérative permet à ses parties prenantes d'exprimer leurs attentes et leurs intérêts et prend en compte ces attentes. Cela suppose également que cette écoute des parties prenantes ne soit pas purement formelle mais qu'elle ait des conséquences sur la fixation des objectifs de la coopérative et sur le choix des moyens mis en œuvre. Mais cela pose également la question de l'arbitrage entre des demandes diverses et parfois conflictuelles.

L'institutionnalisation des organes de représentation des parties prenantes est le moyen nécessaire pour répondre aux exigences sus-mentionnées. Concrètement cela peut prendre des formes relativement conventionnelles pour les parties prenantes non coopérateurs. Par exemple, pour les employés, cela passera par les syndicats, les délégués du personnel, le comité d'entreprise ou l'élection d'administrateurs représentant les salariés. Pour les clients ou les fournisseurs, cela passera par les moyens évoqués précédemment (enquêtes, programmes de fidélisation). Mais cette prise en compte des attentes des diverses parties prenantes pourra aussi passer par une association aux événements davantage tournés vers les coopérateurs: invitation aux assemblées générales des coopérateurs, organisation d'événements festifs et commerciaux, etc.

Indépendamment des organes légaux de représentation des coopérateurs, la principale difficulté est la mise en œuvre de lieux d'expression des coopérateurs. En effet, le principe démocratique ne permet plus le lien direct entre le coopérateur et le conseil d'administration de l'entreprise dès lors que cette dernière atteint une certaine taille. Là encore les pratiques des coopératives démontrent une certaine créativité :

- Découpage de la coopérative en sections et sous-sections, mise en place d'assemblées aux différents niveaux avec dans chaque assemblée la nomination de délégués ou d'administrateurs qui représenteront les coopérateurs à l'assemblée du niveau supérieur. 
- Jumelage des assemblées de coopérateurs avec des événements festifs ou commerciaux pour accroître la participation des coopérateurs aux assemblées.

L'entreprise coopérative ou mutualiste serait ainsi au croisement de la démocratie participative et de la démocratie représentative, ou autrement dit au croisement des attentes de sociétés ou de communautés et de leur participation au processus de gouvernance des organisations. Un modèle complexe, fragile, souvent conflictuel, mais un modèle permettant sans doute de répondre aux défis d'un monde contemporain globalisé et atomisé.

\section{Bibliographie}

Alchian A.A. et Demsetz H. (1972), «Production, Information Costs, and Economic Organization », American Economic Review, vol.62, n5, pp.777-795.

Bachet D. et Naszályi Ph. dir (2011) L'autre finance : existe-t-il des alternatives à la banque capitaliste ?Ed. du Croquant, 390p. Paris, EAN13 : 9782914968973 ISBN : 978-2-914968-97-3

Burnham James (1941), The Managerial Revolution : What is Happening in the World, New York, John Day Company.

Callon Michel, Lascoumes Pierre, Barthe Yannick (2001), Agir dans un monde incertain. Essai sur la démocratie représentative, Seuil, Paris.

Charreaux G. et J.P. Pitol-Belin, 1985, «La théorie contractuelle des organisations : une application au Conseil d'Administration », Economies \& Sociétés, Série Sciences de Gestion, Tome XIX, $n^{\circ}$ 6, juin, p.149-181.

Charreaux G. (1987), « La théorie positive de l'agence: une synthèse de la littérature », in $D e$ nouvelles théories pour gérer l'entreprise, Economica.

Coase R.H. (1937): « The nature of the Firm », Economica, Vol.4, November, pp.331-351.

Collette C. et Pigé B. (2008), Economie sociale et solidaire - Gouvernance et contrôle, Dunod.

Defourny J. (1990), " Démocratie coopérative et efficacité économique - La performance comparée des SCOP françaises », coll. " ouvertures économiques », De Boeck Université.

Desroches H., (1976), «Le projet coopératif, son utopie et sa pratique, ses appareils et ses réseaux, ses espérances et ses déconvenues », Editions Ouvrières, Paris.

Dewey John (2003), Le Public et ses problèmes, Publication de l'université de Pau/Farrago, Paris.

Ebondo E. et Pigé B. (2002), «L'arbitrage entreprise / marché : le rôle du contrôle interne, outil de réduction des coûts de transaction », Comptabilité Contrôle Audit, Vol.8 (2), pp.51-67.

Fama E.F. (1980), «Agency Problems and The Theory of The Firm », Journal of Political Economy, Vol. 88 (2), pp. 288-307. 
Fama E.F. et Jensen M.C. (1983) «Agency Problems and Residual Claims », Journal of Law and Economics, Vol. 26, pp. 327-350.

Galbraith John Kenneth (1967), Le nouvel état industriel, éd. Gallimard.

Gianfaldoni Patrick et Richez-Battesti Nadine (sous la direction de), (2008), «La gouvernance partenariale des banques coopératives françaises », Rapport à la DIIESES (Délégation Interministérielle à l'Innovation, à l'Expérimentation Sociale et à l'Économie Sociale), Université de la Méditerranée et d'Avignon, mars.

Habermas Jürgen (1997), Droit et Démocratie. Entre faits et normes, Gallimard, Paris 1997, 554 pages.

Jensen M.C. et Meckling W.H. (1976), « Theory of the Firm: Managerial Behavior, Agency Costs and Ownership Structure », Journal of Financial Economics, vol.3, octobre, pp.305-360.

Jensen M.C. et Meckling W.H. (1979), « Rights and Production Functions: An Application to Labor-managed Firms and Codetermination », Journal of Business, Vol.52 (4), pp.469-506.

Labye A., Lagoutte C., Renversez F. (2002), « Banques mutualistes et systèmes financiers : une analyse comparative Allemagne, Grande-Bretagne, France », Revue d'Economie Financière, $\mathrm{n}^{\circ}$ 67, pp. 85-109.

Montesquieu Charles-Louis de Secondat (de) (1838), «De l'Esprit des Lois» in Oeuvres complètes, Paris.

Naszályi Ph (2010) Crédit coopératif, histoire et actualité ; l'héritage de Raiffeisen (1818$1888)$ et Schultze-Delitzsch (1808-18885) in Economie sociale et solidaire, Nouvelles trajectoires d'innovations sous la direction de Sophie Boutiller et Sylvain Allemand, collection Marché et Organisation, L'Harmattan, 236 pages,

Naszályi $\mathrm{Ph}$ (2011) La Nouvelle Mutualité ", utopie ou refondation, Essai d'analyse de la gouvernance de la FNIM, (Fédération nationale interprofessionnelle des mutuelles, Editions Universitaires européennes, 548 pages, ISBN : 9786131568916

Pigé B. (2008), Gouvernance Contrôle et Audit des Organisations, Economica.

Pigé B. (2010), Éthique et Gouvernance des Organisations, Economica.

Pigé B. et Paper X. (2009), Normes comptables internationales et gouvernance des entreprises. Le sens des normes IFRS, EMS.

Rancière Jacques (2005), La Haine de la démocratie, La Fabrique, Paris.

Vienney C. (1994), «L'économie sociale », coll. « Repères », La Découverte, Paris.

Williamson O.E. (1975): Markets and Hierarchies, Free Press, New York.

Williamson O.E. (1985): The Economic Institutions of Capitalism, Free Press, New York. 